\title{
The majority of lactococcal plasmids carry a highly related replicon
}

\author{
Jos F. M. L. Seegers, Sierd Bron, Christian M. Franke, Gerard Venema \\ and Rense Kiewiet
}

Author for correspondence: Sierd Bron. Tel: +3150 632105. Fax: +3150632348.

Department of Genetics, Centre of Biological

Sciences, Kerklaan 30, 9715

NN, Haren, The Netherlands

\begin{abstract}
DNA sequence analysis and Southern hybridizations, together with complementation experiments, were used to study relationships between lactococcal plasmid replicons. pWVO2, pWVO4 and pWVO5, which co-exist in Lactococcus lactis subsp. cremoris Wg2, and plL7 (isolated from another strain) all contained a functional replication region which appeared to be very similar to that of some known lactococcal plasmids. They contain a gene encoding a highly conserved RepB protein (60-80\% amino acid identity between pWVO2, pWV04 and pWV05), which is essential for replication. When supplied in trans, repB of pWVO2 complemented a repB deficiency of pWVO5. Upstream of the repB gene, all these plasmids contain a strongly conserved region including a 22 bp sequence tandemly repeated three-and-a-half times, and an AT-rich region. The similarity with pWVO2, which is known to replicate via a theta mechanism, suggests that all plasmids of this family are capable of theta replication. Southern hybridizations revealed that many lactococcal strains contain plasmids of this family.
\end{abstract}

Keywords: lactococcal plasmids, plasmid replication, plasmid incompatibility

\section{INTRODUCTION}

Lactococcal strains generally carry a number of different plasmids, varying in size from approximately $2 \mathrm{~kb}$ to over $100 \mathrm{~kb}$, some of which specify traits of major interest for the dairy industry. A consequence of the plasmid location is that the desired trait is lost when the plasmid is lost from the population. This is a problem of both fundamental and applied interest. Plasmid loss is a frequently observed problem in Gram-positive bacteria, such as Bacillus subtilis (Bron et al., 1991; Gruss \& Ehrlich, 1989) and Staphylococcus aureus (Novick, 1989), and has also been observed in lactococci (Otto et al., 1983). Recently, we showed that the cloning of large DNA fragments in rolling-circle (RC) plasmids results in poor maintenance in Lactococcus lactis (Kiewiet et al., 1993). When the streptococcal theta plasmid pAM $\beta 1$ was used for similar experiments, the resulting recombinant plasmids were, however, stably maintained. Based on this observation, we anticipated that endogenous theta plasmids from lactococci would be valuable for the development of stable (food-grade) cloning vectors in lactic acid bacteria.

Abbreviation: $\mathrm{RC}$, rolling circle.

The EMBL accession numbers for the nucleotide sequences reported in this paper are Z25475 (pIL7), Z25476 (pWVO4) and Z25477 (pWVO5).
One of the possible causes of plasmid loss may be the complex mixture of plasmids in most lactococcal strains. It is conceivable that in strains harbouring related plasmids, interaction at the level of replication or partitioning would result in incompatibility. For future development of stable food-grade plasmid vectors it is, therefore, important to analyse the relationships and interactions between plasmids within one strain. In the present work we analysed the replication regions of a number of lactococcal plasmids. Specifically, we addressed the question whether the various plasmids which co-exist in L. lactis subsp. cremoris $\mathrm{Wg} 2$ are related.

A number of lactococcal plasmids, other than those of the RC-type, have recently been analysed. These plasmids, which show a high degree of similarity, comprise the $8.7 \mathrm{~kb}$ cryptic plasmid pCI305 (Hayes et al., 1991), the lactose plasmid pSK11L (Jahns et al., 1991), the citrate plasmid pSL2 (Horng et al., 1991), the $3.8 \mathrm{~kb}$ cryptic plasmid pWVO2 (Kiewiet et al., 1993), a $28 \mathrm{~kb}$ cryptic plasmid that has been used to construct the nisin-resistant plasmid pVS40 (Von Wright et al., 1990; GenBank accession number L02920), and the bacteriophageresistance plasmid pCI528 (GenBank accession number L06274). One of these, the cryptic plasmid pWVO2, was derived from $L$. lactis subsp. cremoris strain Wg2, which carries a total of five different plasmids (Otto et al., 1983). 
The smallest of these, the RC-type plasmid pWVO1 $(2 \cdot 17 \mathrm{~kb})$, has been studied extensively, and a large set of multipurpose cloning vectors is based on it (Leenhouts $e t$ al., 1991; Kok, 1991). Of the other plasmids from this strain, denoted pWVO2 $(3.8 \mathrm{~kb})$, pWVO3 $(7 \mathrm{~kb})$, pWVO4 (19 kb) and pWVO5 (27 kb), only the first has been studied in some detail. Recently, we showed that pWVO2 replicates via a theta mechanism (Kiewiet et al., 1993). Hitherto, no sequence data on the replication regions of pWVO3, pWVO4 and pWVO5 have been available. pWVO5 is of particular interest, since it carries the gene specifying the proteinase required for casein breakdown in cheese production (Otto et al., 1983; Kok et al., 1988). Loss of proteinase activity is frequently observed during cheese production and appears to result from the loss of a plasmid specifying the proteinase gene (Otto et al., 1983).

In this paper we describe the isolation and characterization of functional replication regions of pWVO4, pWVO5 and pIL7. The latter plasmid, extracted from L. lactis IL594, specifies a restriction/modification system that renders carrier strains resistant to a number of bacteriophages (Chopin et al., 1984). We were unable to clone the replication functions of pWVO3, and this plasmid was therefore not included in these studies. Since this type of plasmid is closely related to the theta-replicating plasmid pWVO2 (Kiewiet $e$ t al., 1993), this family of plasmids is likely to be valuable for the development of stable, foodgrade cloning vectors, and for studies on the mechanisms underlying plasmid instability and (in)compatibility in lactococci.

\section{METHODS}

Bacterial strains, growth conditions and plasmids. All strains and plasmids used in this study are listed in Table 1. Eschericbia coli cells were transformed as described by Mandel \& Higa (1970) and plated on LB agar or grown on LB medium (Sambrook et al., 1989), supplemented with $100 \mu \mathrm{g}$ erythromycin $\mathrm{ml}^{-1}$ or $75 \mu \mathrm{g}$ ampicillin $\mathrm{ml}^{-1}$ when required. Tests for replication proficiency of plasmid derivatives were carried out in L. lactis subsp. lactis MG1363 (Gasson, 1983). Cells, grown on M17 medium (Terzaghi \& Sandine, 1975), were transformed by electroporation (Holo \& Nes, 1989) and plated on M17 agar, containing $0.5 \mathrm{M}$ sucrose and $5 \mu \mathrm{g}$ erythromycin $\mathrm{ml}^{-1}$.

Isolation of plasmid DNA; DNA manipulations. Isolation of plasmid DNA by the alkaline lysis method was essentially as described by Sambrook et al. (1989) with some minor modifications. Complete lysis of cells was achieved by resuspending cell pellets in solution I supplemented with lysozyme in a minimal volume of $7 \cdot 5 \%$ of the original volume. This step was followed by an incubation at $55^{\circ} \mathrm{C}$ for $10-15 \mathrm{~min}$. For largescale plasmid isolations, DNA was precipitated with 2-propanol prior to phenol/chloroform extraction in order to reduce the volume. Plasmids were isolated from $1 \%(\mathrm{w} / \mathrm{v})$ agarose gels using the Prep-a-gene kit as recommended by the manufacturer (BioRad). DNA sequencing was performed by the dideoxynucleotide chain-termination method of Sanger et al. (1977). All sequences were determined on both strands. PCR reactions were carried out as described by Sambrook et al. (1989). Deletions for determining the region required for replication of
pJR7 were made with Exo III and mung bean nuclease as described by the supplier (Stratagene).

Oligonucleotides. To sequence the replication regions of pWVO4, pWVO5 and pIL7 a set of ten oligonucleotides was designed. The sequence of these was based on homologous regions between the nucleotide sequences of the rep genes of pWVO2, pCI305, pSL2 and pSK11L. Oligonucleotides were constructed in such a way that at locations where different nucleotides were present between the different sequences, these different nucleotides could be incorporated (indicated in parentheses in Table 2), resulting in a mixture of different oligonucleotides in equal amounts. Oligonucleotides P1 through P5 were designed to prime DNA synthesis in one direction; the partly complementary oligonucleotides P6 through P10 were designed to prime DNA synthesis in the other direction. These sets of oligonucleotides enabled us to obtain the sequences for both strands. The position of these primers, relative to the amino acid sequences encoded by the rep genes, is indicated in Fig. 1.

Regions outside the rep gene were sequenced with oligonucleotides based on the sequences obtained from oligonucleotides P1 through P10 and completed for both strands.

Southern hybridizations. Southern transfers were carried out as described by Sambrook et al. (1989) using Gene Screen Plus membranes as carrier (NEN-Research Laboratories). DNA fragments used as probes were prepared by PCR of complementary DNA using primers 1 and 10 (Table 2) and pWVO2 as a template. Probe labelling, hybridization and the detection of complementary DNA were conducted using the ECL gene detection system (Amersham) as recommended by the manufacturer. Hybridization conditions were as described for homologous probes $\left(42^{\circ} \mathrm{C}, 0.5 \mathrm{M} \mathrm{NaCl}\right)$.

\section{RESULTS}

\section{Cloning of replication regions of plasmids pWVO4, pWVO5 and pIL7}

Plasmids pWVO4 and pWVO5 were isolated from agarose gels on which the complex plasmid mixture of $L$. lactis subsp. cremoris $\mathrm{Wg} 2$ was separated. pIL7, originally present in L. lactis IL594, was isolated from a lactococcal strain carrying only this plasmid (Chopin et al., 1984). Different approaches were used for the cloning of the replication regions of the various plasmids that were compared in this study.

pWV04/pJRO4. Plasmid pWVO4 was digested with $B g / I$. This resulted in three large fragments which were cloned into the $B g / \mathrm{II}$ site of pMTL23E (Table 1). Since pMTL23E cannot replicate in $L$. lactis, this vector was used to screen for DNA fragments that enabled replication in this bacterium. $\mathrm{Em}^{\mathbf{R}}$ transformants were initially selected in E. coli JM101. Only recombinant plasmids which carried the $4.5 \mathrm{~kb} \mathrm{Bg} / \mathrm{II}$ fragment from pWVO4, in either orientation, were able to support replication after subsequent transfer to L. lactis. One of these plasmids, pJRO4 (Table 1), was used for further analysis.

pWVO5/pJRO5. Plasmid pWVO5 was digested with EcoRI. The resulting seven fragments were inserted into the EcoRI site of pMTL23E. The ligation mixture was used to transform $L$. lactis directly. Transformants 
Table 1. Bacterial strains and plasmids

\begin{tabular}{|c|c|c|}
\hline $\begin{array}{l}\text { Bacterial strain } \\
\text { or plasmid }\end{array}$ & Properties & $\begin{array}{l}\text { Source or } \\
\text { reference }\end{array}$ \\
\hline \multicolumn{3}{|l|}{ E. coli } \\
\hline JM101 & $\begin{array}{l}\text { sup } \mathrm{E} \text { this(lac-pro } \mathrm{AB})\left[\mathrm{F}^{\prime} \text { tra } \mathrm{D} 36 \text { pro } \mathrm{AB} \text { lac } \mathrm{I}^{q}\right. \\
\text { lac } \mathrm{Z} \Delta \mathrm{M} 15\end{array}$ & $\begin{array}{l}\text { Yanisch-Perron et al. } \\
\text { (1985) }\end{array}$ \\
\hline \multicolumn{3}{|c|}{ (1) - } \\
\hline MG1363 subsp. lactis & Plasmid-free, $\mathrm{Lac}^{-} \mathrm{Prt}^{-}$ & Lab. collection \\
\hline subsp. cremoris Wg2 & Industrial strain, containing pWVO1-pWVO5 & Lab. collection \\
\hline subsp. cremoris E8L & Natural strain, cryptic plasmid complement & Visser et al. (1986) \\
\hline subsp. cremoris $\mathrm{H} 61$ & Natural strain, cryptic plasmid complement & Okamoto et al. (1985) \\
\hline subsp. cremoris F16 & Natural strain, cryptic plasmid complement & Okamoto et al. (1983) \\
\hline subsp. cremoris $\mathrm{Hpl}$ & Natural strain, cryptic plasmid complement & Exterkate (1975) \\
\hline subsp. cremoris 916 & Natural strain, cryptic plasmid complement & Lab. collection \\
\hline subsp. cremoris $\mathrm{Cla} 2$ & Natural strain, cryptic plasmid complement & Lab. collection \\
\hline subsp. cremoris 109 & Natural strain, cryptic plasmid complement & Lab. collection \\
\hline L10 & Natural strain, cryptic plasmid complement & Lab. collection \\
\hline P8-2-47 & Natural strain, cryptic plasmid complement & Lab. collection \\
\hline $9 \mathrm{~B} 4$ & Natural strain, cryptic plasmid complement & Lab. collection \\
\hline \multicolumn{3}{|l|}{ Plasmids } \\
\hline pWVO2 & $3.8 \mathrm{~kb}$, cryptic, from L. lactis $\mathrm{Wg} 2$ & Otto et al. (1983) \\
\hline pWVO4 & $6.5 \mathrm{~kb}$, cryptic, from L. lactis Wg2 & Otto et al. (1983) \\
\hline pWVO5 & $27 \mathrm{~kb}$, specifies proteinase, from L. lactis $\mathrm{Wg} 2$ & Otto et al. (1983) \\
\hline $\mathrm{pIL} 7$ & $\begin{array}{l}31 \mathrm{~kb} \text {, specifies restriction/modification system, from } \\
\text { L. lactis IL594 }\end{array}$ & Chopin et al. (1984) \\
\hline pMTL23E & $\begin{array}{l}\mathrm{Ap}^{\mathrm{R}} \mathrm{Em}^{\mathrm{R}}, 4 \cdot 0 \mathrm{~kb}, \mathrm{pMTL} 23 \text { derivative containing the } \\
\mathrm{Em}^{\mathrm{R}} \text { gene in the ClaI site (pE194cop6 coordinates } \\
3140-1939) \text { and the T1T2 transcriptional terminator } \\
(550 \mathrm{bp} \text { ) in the EcoRI site; not able to replicate in } \\
\text { L. lactis }\end{array}$ & Kiewiet et al. (1993) \\
\hline pJRO4 & $\begin{array}{l}\text { pMTL23E, carrying a } 4.5 \mathrm{~kb} B g / \mathrm{II} \text { fragment of } \\
\mathrm{pWVO} 4 \text {, capable of autonomous replication in } \\
\text { L. lactis }\end{array}$ & This work \\
\hline pJRO5 & $\begin{array}{l}\text { pBSKII-, carrying a } 7 \mathrm{~kb} \text { EcoRI fragment of } \\
\text { pWVO5, capable of autonomous replication in } \\
\text { L. lactis }\end{array}$ & This work \\
\hline pJRO55 & $\begin{array}{l}\text { pMTL23E, carrying a } 3.9 \mathrm{~kb} \text { HindIII fragment of } \\
\text { pWVO5, not capable of autonomous replication in } \\
\text { L. lactis }\end{array}$ & This work \\
\hline $\mathrm{pJR} 7$ & $\begin{array}{l}\text { pMTL23E, carrying a } 3.2 \mathrm{~kb} \text { Sau3A fragment of } \\
\text { pIL7, capable of autonomous replication in L. lactis }\end{array}$ & This work \\
\hline
\end{tabular}

selected on erythromycin-containing plates were analysed for their plasmid content. All appeared to contain the same $7 \mathrm{~kb}$ EcoRI insert, which was found in both orientations. Plasmids containing this insert in one orientation, pJRO5 (Table 1) were used for further analysis.

plL7/pJR7. In an experiment aimed at the cloning of fragments from large (theta-replicating) plasmids that would increase segregational plasmid stability, a $3.2 \mathrm{~kb}$ Sau3A fragment of pIL 7 was isolated from a partial Sau3A digest. This fragment was subsequently cloned into the Bam HI site of pMTL23E. Plasmid DNA containing this insert was isolated from $E$. coli and used to transform $L$. lactis. Plasmid-containing transformants were obtained, indicating that the $3.2 \mathrm{~kb}$ insert carried a region of pIL7 that was capable of sustaining autonomous replication in L. lactis. This plasmid, PJR7 (Table 1), was used for further analysis.

\section{DNA sequences of the replication regions of pJRO4, pJRO5 and pJR7}

Based on the similarities between pWVO2 and several other previously described lactococcal plasmids (Hayes et al., 1991; Jahns et al., 1991 ; Horng et al., 1991 ; Kiewiet et al., 1993) we speculated that most lactococcal plasmids, other than those of the RC-type, might contain highly related, functional theta-type replication regions (Kiewiet et al., 1993). Support for this idea was obtained from 
Table 2. Oligonucleotides used for sequencing of replication genes firom pWVO4, pWVO5 and pIL7

Nucleotides in parentheses indicate different possibilities at that location.

\begin{tabular}{|c|c|c|c|c|}
\hline \multirow[t]{2}{*}{ Oligo } & \multirow[t]{2}{*}{ Sequence } & \multicolumn{3}{|c|}{$\begin{array}{c}\text { Readable sequence } \\
\text { obtained } \\
\text { in the dideoxy } \\
\text { chain-termination reaction }\end{array}$} \\
\hline & & pWVO4 & pWVO5 & pIL7 \\
\hline P1 & $\mathrm{AA}(\mathrm{TA}) \mathrm{CAA} A \mathrm{AG}$ CAG GTG C & - & - & + \\
\hline P2 & ATG CAA (GA)A(GA) CAA GC(CT) TTT T & - & + & + \\
\hline P3 & GAA (TC)TA (TC)AA CCA ATA CG & - & - & + \\
\hline P4 & (CT)AT TGT CTT TCA TAT & + & + & + \\
\hline P5 & GG(GT) GTC AAA GAC CAC TTG TC & + & + & + \\
\hline P6 & GAC AAG TGG TCT TTG AC(AC) CC & + & + & + \\
\hline P7 & (GA)AT ATG AAA GAC AAT (GA) & + & + & + \\
\hline P8 & CGT ATT GGT T(AG)T A(AG)T TC & - & + & + \\
\hline P9 & AAA A(GA)G CTT G(CT)T (CT)TT GCA T & + & + & + \\
\hline P10 & CAA GGT (TCA) (TA) (GA) CAC CTG CTT TT & + & + & + \\
\hline
\end{tabular}

Southern hybridizations in which the $\operatorname{rep} B$ gene of pWVO2 was used as a probe against the entire plasmid content of $L$. lactis subsp. cremoris $\mathrm{Wg} 2$. This resulted in cross-hybridizations with $\mathrm{pWVO} 2$ as well as pWVO3, pWVO4 and pWVO5 (results not shown). To study whether $\mathrm{pJRO} 4$ and $\mathrm{pJRO5}$, which were obtained from pWVO4 and pWVO5, as well as pJR7, contained replication regions similar to that of $\mathrm{pWVO} 2$, a set of ten oligonucleotide primers (Table 2) was designed on the basis of conserved regions in the nucleotide sequences of the rep genes of pCI305, pSK11L, pSL2 and pWVO2. With most of the oligonucleotides, clear sequence ladders were obtained (Table 2). To complete the sequence for both strands, additional oligonucleotides, based on the sequences obtained, were used. Analysis of the resulting sequences in each case revealed an ORF, similar to that of the rep genes of the plasmids from which the oligonucleotides were designed. Insertion of random $\lambda$ DNA fragments into the SnaBI site in this ORF of pJRO4, or the HindIII site in the corresponding ORF of pJRO5, abolished replication in L. lactis. The minimal region required for replication of pJR7 was determined by creating deletions from either side of the cloned fragment with Exo III and mung bean nuclease and subsequent transformation to L. lactis. Deletions in one direction were created using the $\operatorname{Sac} \mathrm{I}$ site as the $3^{\prime}$ protected end and the Eco47-3 site as the 5' starting point for Exo III. Deletions in the other direction were created with the DraIII site as the $3^{\prime}$ protected end and the $M l u \mathrm{I}$ site as the $5^{\prime}$ starting point. The deletion end-points and the entire upstream region of the pIL7-derived ORF of $\mathrm{pJR} 7$ were sequenced using the M13 universal and reverse primers (Short et al., 1988). Replication of pJR7 in $L$. lactis was abolished when deletions removed the last 153 nucleotides from this ORF. These results show that the respective ORF in each of the analysed replicons is required for replication.
By analogy with similar regions of pCI305, pSK11L, pSL2 and pWVO2, the described ORFs of pJRO4, pJRO5 and pJR7 were denoted repB. In Fig. 1 the deduced amino acid sequences of the RepB proteins of pWVO2, pWVO4, pWVO5 (from strain Wg2) and pIL7 (from strain IL594) are compared with those of the equivalent proteins of other replicons. The sequences show an overall identity of approximately $30 \%$. Conservation was extremely high in the $\mathrm{N}$-terminal and central regions. The levels of identity are even higher for plasmids of certain subgroups. For instance, the levels of identity amount to $60-80 \%$ for the RepB proteins of pWVO2, pWVO4 and pWVO5, which are derived from the same strain.

Additional sequences of the region upstream of $r e p B$ of $\mathrm{pWVO} 4$ and $\mathrm{pWVO5}$ were determined by primer walking using oligonucleotides based on the sequences obtained. The sequence of this region of $\mathrm{pJR} 7$ could be determined with universal and reverse primer by using deletion derivatives, obtained from the Exo III and mung bean nuclease reactions. The corresponding regions of $\mathrm{pWVO} 2$ (Kiewiet et al., 1993) and pCI305 (Hayes et al., 1991) have previously been shown to be required for replication. Sequences similar to those determined for the previously mentioned lactococcal plasmids were found (Fig. 2). Both contain a 22 bp sequence tandemly repeated three-and-ahalf times. Comparison of all repeats revealed a consensus sequence, reading $5^{\prime}$ ANN $(G / C)(T / C) N(T / A) N A A A A-$ AAC $(T / G)(G / A) T G T A T-3$ '. In addition, two regions of dyad symmetry, overlapping the putative promoter region, were identified. The inverted repeat downstream of the -10 region is highly conserved in all three $\mathrm{pWVO} 2$-related plasmids of $L$. lactis subsp. cremoris $\mathrm{Wg} 2$, as well as in most other lactococcal plasmids described. The region upstream of the $22 \mathrm{bp}$ repeats carries a number of AT-rich stretches separated by GC-clusters. The 


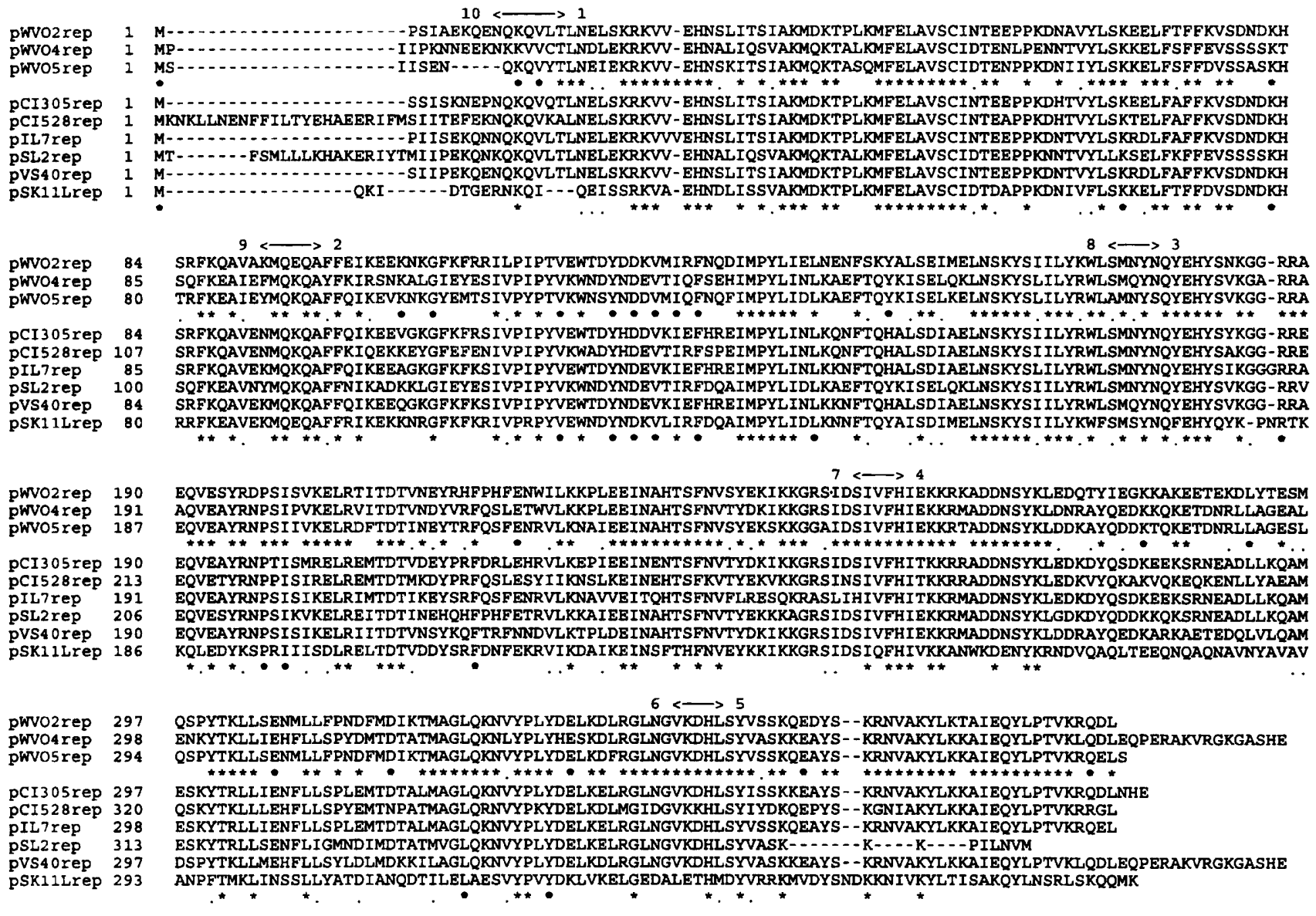

Fig. 1. Alignment of deduced amino acid sequences of RepB proteins specified by lactococcal plasmids pWVO2 (Kiewiet et al., 1993), pWVO4, pWVO5, pCl305 (Hayes et al., 1991), pCl528 (GenBank accession number L06274), plL7, pSL2 (Horng et al., 1991), pVS40 (GenBank accession number L02920) and pSK11L (Jahns et al., 1991). The one-letter code is used. Numbers to the left of the sequences indicate the position of the first ( $\mathrm{N}$-terminal) aa residue of each protein. The first three plasmids (pWVO2, pWVO4 and pWVO5) were derived from the same strain (L. lactis subsp. cremoris Wg2). Identical amino acids in all sequences are indicated with an asterisk, similar amino acids with a dot. The relative positions of the primers listed in Table 2 are indicated above the sequence. Directions for primer extension are indicated by arrowheads.

nucleotide sequences of these stretches show a very high level of conservation (Fig. 2). As for pWVO2 and pCI305, this region was determined to be part of the minimal region required for replication of pJR7. Replication of derivatives obtained from the Exo III and mung bean nuclease reaction was abolished when deletions extended within this region. The smallest derivative that still supported replication extended $134 \mathrm{bp}$ upstream of the region as represented in Fig. 2.

\section{Presence of an additional ORF downstream of repB}

Immediately downstream of the $r e p B$ gene, the start of another ORF could be detected in the sequences of pJRO4, pJRO5 and pJR7. The entire sequence of this ORF was determined for pJR7 and found to encode a putative protein of 208 amino acids (Fig. 3a). The putative start codon (ATG) of this ORF, denoted ORF X, overlaps with the stop codon (TGA) of the upstream repB gene, which is indicative of translational coupling between these genes. A similar start can be identified in the related
ORF $\mathrm{X}$ sequences of most other lactococcal plasmids (Fig. 3b). In the sequence of pWVO2 only the first 14 codons of ORF $\mathrm{X}$ are present. The start of ORF $\mathrm{X}$ in all the plasmids analysed is preceded by an appropriate Shine-Dalgarno sequence (Van de Guchte et al., 1992). All deduced amino acid sequences were highly similar and showed an $\alpha$-helix-turn- $\alpha$-helix motif between amino acid residues 6 and 28, indicating a DNA-binding potential for this region. Deletion of this ORF from pJR7 by the Exo III and mung bean nuclease method did not affect replication. No putative transcriptional terminator could be identified downstream of either $r e p B$ or ORF X. The observation that ORF X can be deleted from $\mathrm{pJR} 7$ without affecting replication, and the fact that this ORF is almost completely absent from pWVO2, suggest that it is not essential for replication.

\section{repB of pWVO2 can complement repB of pWVO5}

A $3.9 \mathrm{~kb}$ HindIII fragment of pJRO5, contained entirely within the $7 \mathrm{~kb}$ EcoRI fragment of pWVO5, which carried 


$$
\star \star \star
$$

\begin{tabular}{|c|c|c|}
\hline pwoO2 & ACAAGTTTTAAAGCCTAT-ATCT & ATTTATCAT-ATATATTTTAATCTTTT \\
\hline WVO4 & AAAAAAAGCGAAGCCTAT-ATCT & ATTTATCAT-ATATATTTTAATCTTTT \\
\hline & AAAAAGCGAAGCCTATTATAT & СTTTT \\
\hline & AAAAAGGCGAAGC-TATTATAT & ATTTATCAT-ATATATTTTAA \\
\hline & AAAATAGCGAAGCCTATTATAT & ATTTATCTT-ATATATTTTAATCTTTT \\
\hline & AAAAAAAGCGAAGCCTAT - ATTA & ATTTATCAT-ATATATTTTAATCTTTT \\
\hline & AAAAAAGGCGAAGCCST & ATTTATCAT-ATATATTTTAATCTTTT \\
\hline & AAAAAAGGCGAAGCCTATTATAT & 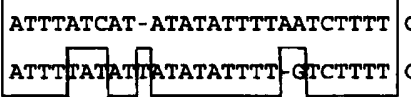 \\
\hline
\end{tabular}

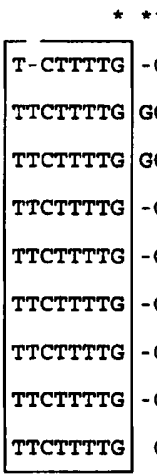

$\star \star \star \star$

- CGTGAAAAAAAA-GTCAGTGGTAGCAAGgGTATGCAGAATTAAAC GCGTGCTAAAAAAAATCAGTGTTTTCAAGgGTATTAAGATTTTTAT GCGTGAAAAAAAAAGTCAGTGGTAGCAAGGGAGGACAGATTTTTAT - CGTGAAAAAAAA-GTCAGTGTTTATAGgCTTATCCAAATTTTTAT CGTCAAAAAAAAA-TCAATATTTTCAAGGCTTTATAGAATTATAT CGTCAAAAAAAA-GTTAGTATTTTTAAGGGGTTACAGAATAATAT CGTGGAAAAAAA-GTCAGTGTTTAAAGGTGGATACAGAATTATAG CGTGAAAAAAAA-GGCAGTGTTTTCGCTAGTTATAGAAATTAAAC CGAAAAAAAAAAATCTAGTGTTTGCAAGGGGTAACAGGATTATAG

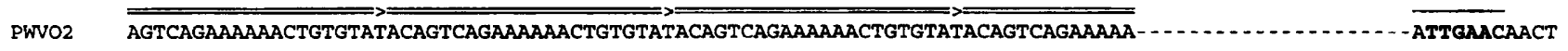

PWVO4 AGCAAGAAAATCGATGTATATAGCAAGAAAAATCGATGTATATAGCAAGAAAAATCGATGTATATAGCAAGAAAAA $\ldots \ldots$

PWVO5 AGCAACAAAAAACGATGTATATAGCAACAAAAAACGATGTATATAGCAACAAAAAACGATGTATATAGCAACAAAAA $\ldots \ldots \ldots$

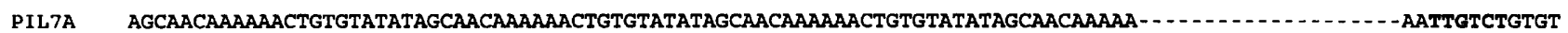

PCI305 ACCAACAAAAAACTGTGTATATACCAACAAAAAACTGTGCATACACCAACAAAAAACTGTGCATATACCAA $\ldots \ldots$

PCI528 AGCATAAAAAAACTGTGTATATAGCATAAAAAAACTGTGTATATAGCATAAAAAAACTGTGTATATAGCATAAAAAA - $\ldots$

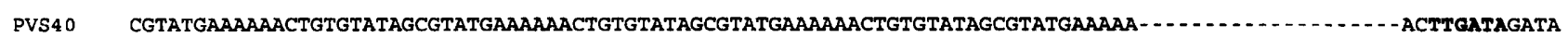

PSL2 AGTCACAAAAATCGATGTATACAGTCACAAAAATCGATTTTTGTGACTCTATGCATCGATGTATACGAGTCACAAAAATCGATGTACACAGTACGACTTTTGTATTTGT

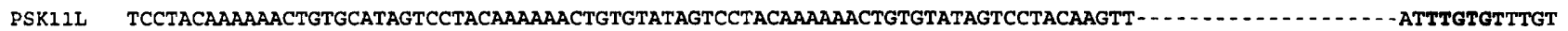

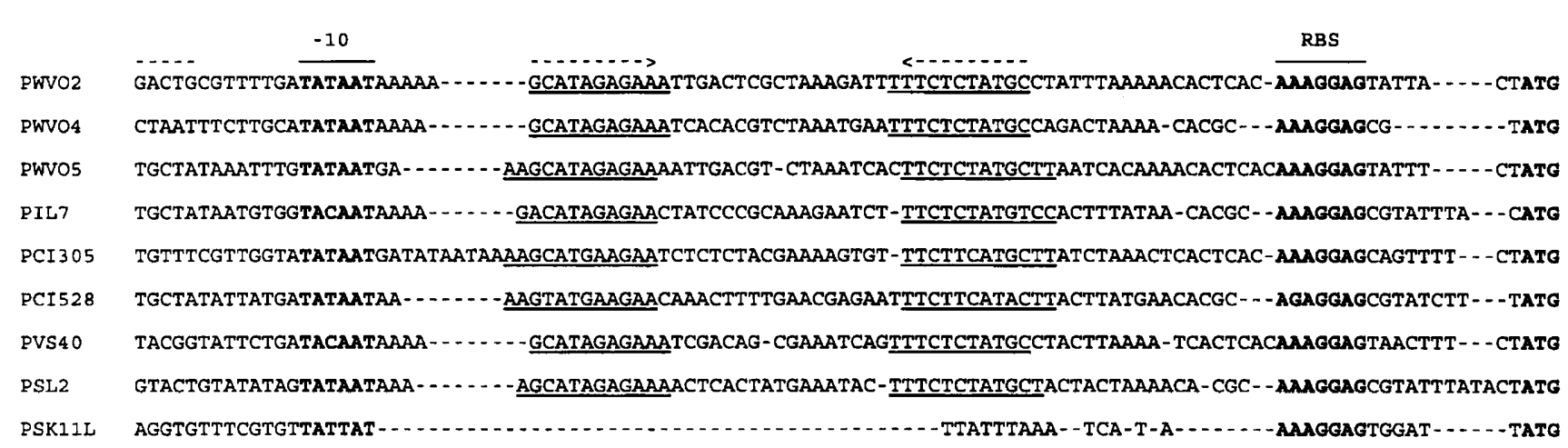

Fig. 2. Alignment of the region upstream of repB of the plasmids described in Fig. 1. Regions with strong sequence conservation are boxed. GC-regions, flanking AT-rich regions, are indicated with asterisks. The 22 bp sequences repeated three-and-a-half times are indicated by double arrows. Inverted repeat regions are indicated by dashed arrows. Putative transcription and translation signals are indicated in bold and by single lines.

the region upstream of the $r e p B$ gene and the first 85 codons of the $r e p B$ gene, was cloned into the HindIII site of pMTL23E and propagated in E. coli. This upstream region is essential for replication of pCI305 (Hayes et al., 1991) and pWVO2 (Kiewiet et al., 1993) as well as pJR7. The resulting plasmid, denoted pJRO55, was no longer capable of autonomous replication in L. lactis. pJRO55 was then used to transform an L. lactis strain carrying only pWVO2. As a control, pJRO5 was used to transform $\mathrm{pWVO} 2$-containing $L$. lactis cells. Transformants were selected on M17 agar supplemented with erythromycin. The transformation efficiency with pJRO55 was about 10fold lower than with PJRO5. Several erythromycinresistant transformants were analysed for their plasmid content. pJRO5 could clearly be detected on agarose gels in small-scale plasmid isolations $(1.5 \mathrm{ml}$ culture) of $L$. lactis, while pJRO55 could only be detected using largescale plasmid isolations (100 ml culture). On the basis of ethidium bromide fluorescence intensities on agarose gels we estimated the copy number of pJRO55 in these cells to be less than one per chromosome equivalent. To verify that plasmid pJRO55 was intact and that $\mathrm{Em}^{\mathrm{R}}$ transformants were not the result of recombination between pWVO2 and pJRO55, plasmid DNA from several erythromycin-resistant transformants was isolated and used to transform $E$. coli. Ten of the transformants were analysed for their plasmid content. All appeared to contain intact $\mathrm{pJRO}$ 55. These results indicate that the $\operatorname{rep} B$ gene of $\mathrm{pWVO} 2$, when supplied in trans, can support the replication of $\mathrm{pJRO}$ 55, which lacks a functional copy of its own $r e p B$ gene. In addition, this experiment provides further proof that the upstream region of $\operatorname{rep} B$ is required 
(a)

ORF X PIL7

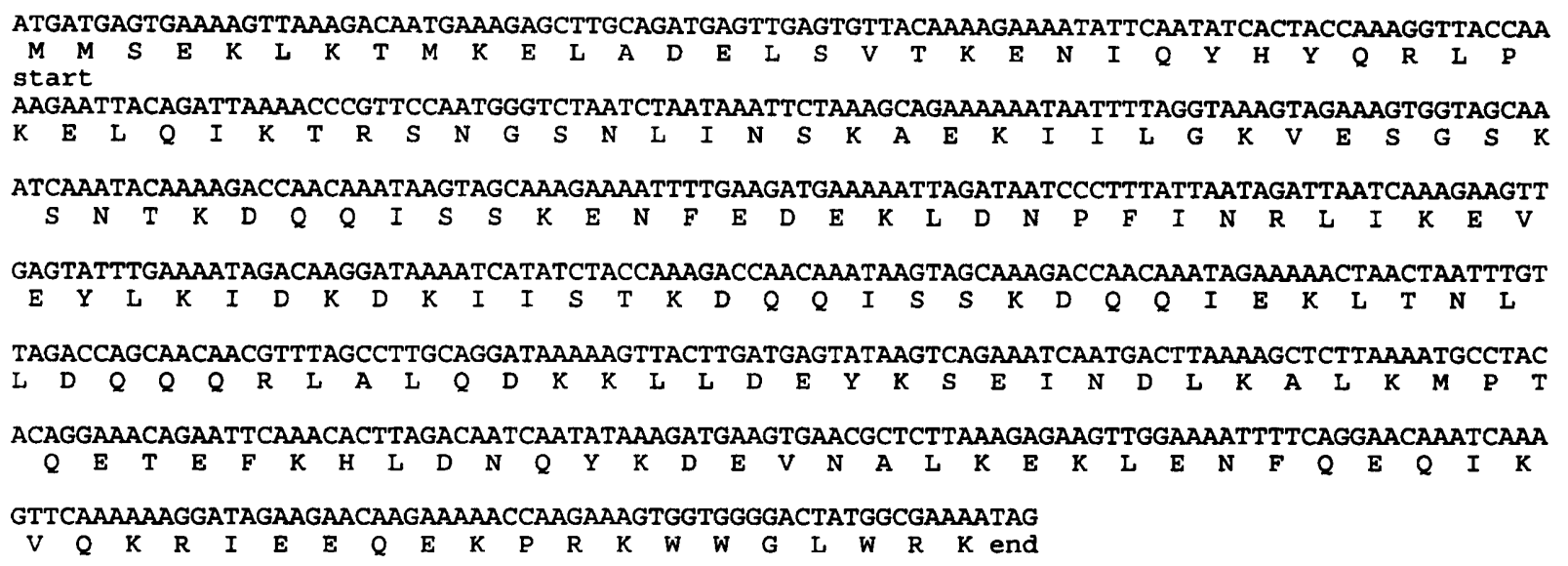

(b)

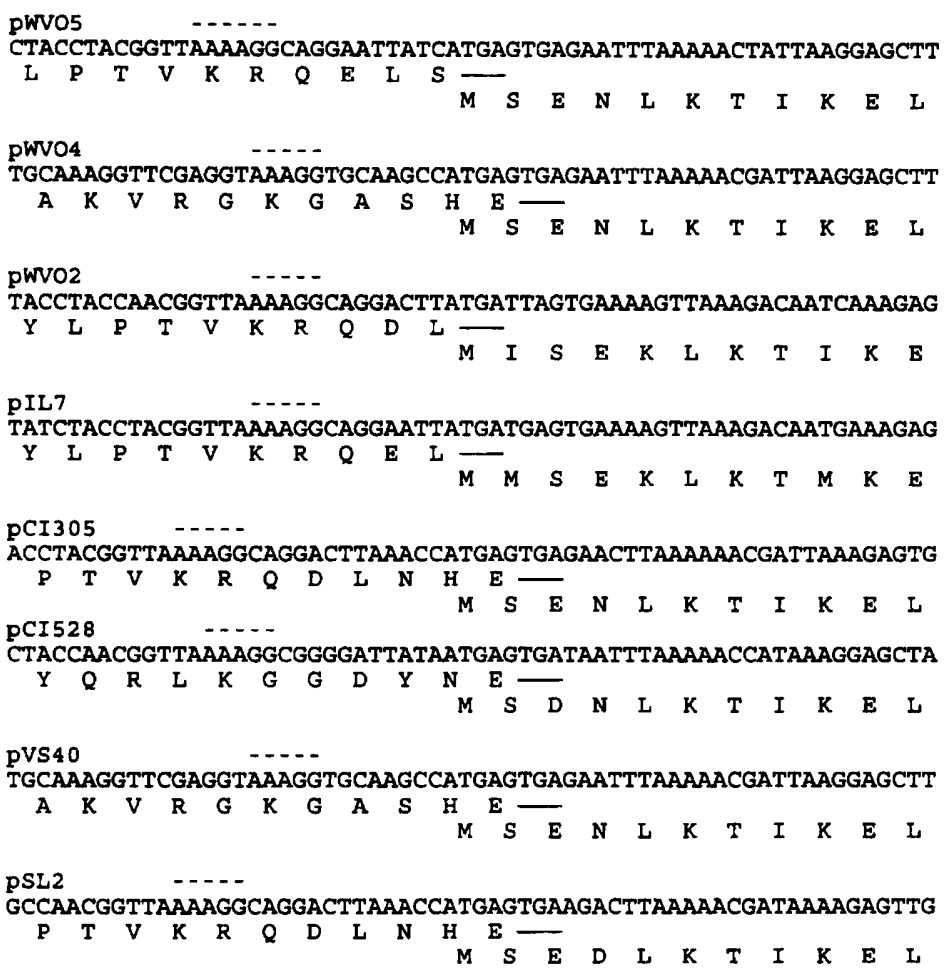

Fig. 3. (a) Complete nucleotide sequence of ORF $X$ of plL7. The deduced amino acid sequence is presented below the sequence in the single-letter code. (b) Probable translational coupling between the $\operatorname{rep} B$ genes of eight plasmids and ORF $X$. The putative Shine-Dalgarno sequence is indicated by a dashed line above the sequence. The stop codon of the rep gene is indicated with a solid line below the sequence. ORF $X$ of pWVO2 ends after the 14 th codon. From the nucleotide sequence of $\mathrm{PSL2}$, as obtained from GenBank (accession number $X 56550$ ), the ORF encoding $r e p B$ ends at the position indicated in Fig. 1. Deletion of a single base in the $3^{\prime}$ region of the nucleotide sequence of this gene, however, would extend the ORF to the location indicated here. In that case, ORF $X$ would also be translationally coupled to the rep $B$ gene. for replication. In the absence of selective pressure pJRO55 was rapidly lost from the culture, whereas pJRO5 was stably maintained. Loss of $\mathrm{pWVO} 2$ was never observed.

\section{Southern hybridizations of plasmids from various lactococcal strains}

Hayes et al. (1991) showed that the type of replicon described here is widespread among lactococci. We tested an additional set of ten $L$. lactis strains for the presence of plasmids with a similar replicon. A $1 \mathrm{~kb}$ PCR fragment of the replication gene $r p B$ of $\mathrm{pWVO} 2$, obtained with primers 1 and 6 (Table 2), was used as a probe. At least one plasmid in each strain tested was found to hybridize to this fragment (Fig. 4).

\section{DISCUSSION}

In the present work we have studied relationships between lactococcal plasmids which are not of the RC type. The most significant conclusions are: (1) that many of these plasmids contain a functional replication region that is part of a family of highly related replicons; and (2) that different plasmids containing these replication regions coexist in one and the same Lactococcus strain. We showed that at least three out of five different plasmids present in $L$. lactis subsp. cremoris $\mathrm{Wg} 2$ contain a functional replication region of this family. One of these plasmids 
(a)

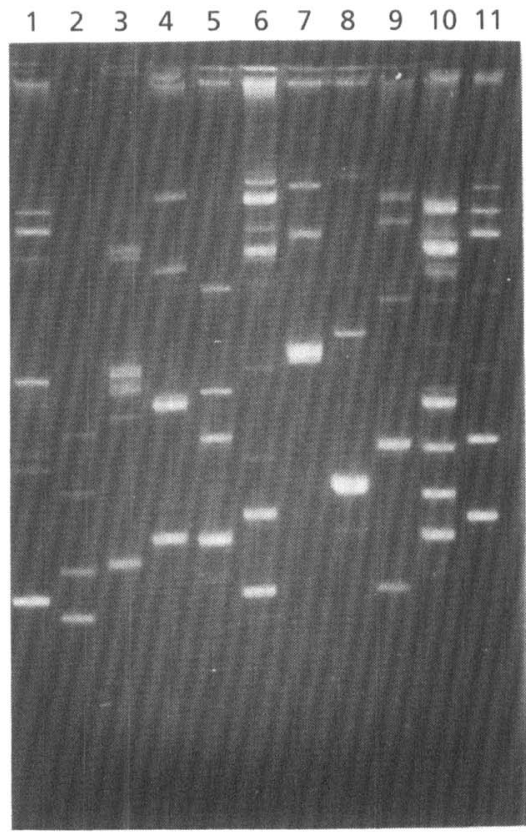

(b)

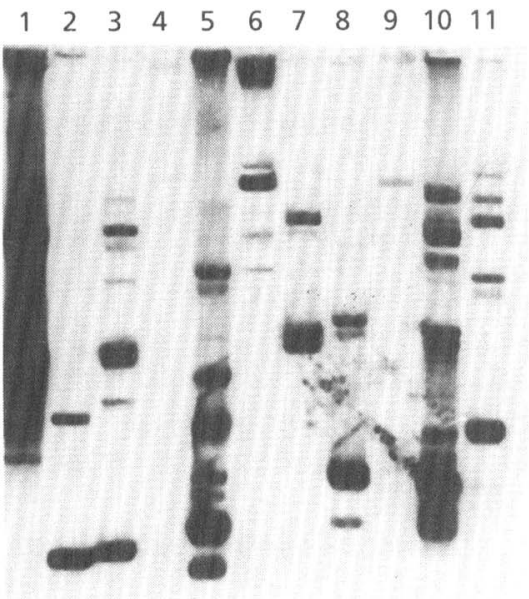

Fig. 4. Distribution of the repB-like replication gene in a number of lactococcal strains. (a) Plasmid profiles of a number of natural Lactococcus strains: 1 , L. lactis subsp. cremoris Wg2; 2 , L. lactis subsp. cremoris H61; 3, L. lactis subsp. cremoris F16; 4, L. lactis subsp. cremoris Hpl; 5 , L. lactis subsp. cremoris E8L; 6 , L. lactis subsp. cremoris Cla2; 7, L. lactis subsp. cremoris $109 ; 8$, L. lactis subsp. lactis L10; 9, L. lactis subsp. cremoris $916 ; 10$, L. lactis subsp. lactis P8-2-47; 11, L. lactis subsp. lactis 9B4. (b) Fluorograph of Southern hybridization of the DNA in (a) with the $1 \mathrm{~kb}$ PCR fragment obtained with primers 1 and 6 of the repB gene of pWVO2.

(pWVO2; Kiewiet et al., 1993) has been shown to replicate via a theta mechanism. This suggests that the other members of this plasmid family can replicate via a similar mechanism. The results obtained do not rule out the possibility that additional functional replication regions might exist on pWVO4, pWVO5 or pIL7. We consider this possibility unlikely, however, since with none of the restriction digests used here (including a partial Sau3A digest of pIL7) were alternative replicons observed.

The replicons studied in this work, and other plasmids of this family, can be divided in two segments. One segment contains the $r e p B$ gene, encoding the $\mathrm{RepB}$ protein, which is essential for replication of pCI305 (Hayes et al., 1991) and pWVO2 (Kiewiet et al., 1993). The three plasmids analysed in the present studies also require an intact repB gene for replication. As shown for pCI305 and pWVO2, the other essential segment is the non-coding region (approximately $300 \mathrm{bp}$ ) upstream of repB. By analogy with Gram-negative theta-replicating plasmids, like RSF1010 (Frey et al., 1992) and RK2 (Perri et al., 1991), for which it has been established that a number of direct repeats are required for binding of the Rep protein, it is conceivable that the 22 bp sequence repeated three-and-ahalf times also constitutes the binding region for the RepB protein in theta-replicating lactococcal plasmids and that this binding is required for initiation of replication. In this context, the observation that $\mathrm{pWVO} 2$ can complement a RepB deficiency of pJRO5 is of interest. This can be explained by assuming that RepB of $\mathrm{pWVO} 2$ can recog- nize the binding region of $\mathrm{pJRO} 55$ and initiate replication. Since RepB is also required for replication of $\mathrm{pWVO} 2$ and probably has a higher affinity for its cognate binding sequence, $\mathrm{pJRO} 55$ will be maintained at a much lower level and rapidly be lost in the absence of selective pressure. Differences in the sequence of the $22 \mathrm{bp}$ repeats of plasmids of this type are likely to determine the difference in affinity of RepB. This may explain why pWVO2 and pJRO5, which carry related repB genes and related 22 bp repeats, can co-exist within one host strain. Affinity studies, currently in progress, between the replication proteins from this family of replicons with the different repeated sequences should give more insight into the phenomenon of plasmid (in)compatibility within one strain.

The region upstream of the $22 \mathrm{bp}$ repeats is highly conserved. Deletion analysis of $\mathrm{pWVO} 2$ has shown that this region is required for replication (Kiewiet et al., 1993). On the basis of its high AT content, this region could be the site of strand separation from which replication starts. This does not, however, require strong conservation of the nucleotide sequence. Possibly, this region is the recognition site for host-encoded functions involved in replication, such as DnaB- and DnaC-like proteins, which are known to be involved in the replication initiation of a number of theta-replicating $E$. coli plasmids (Bramhill \& Kornberg, 1988).

The function of ORF $X$, the expression of which is probably translationally coupled to that of $\operatorname{rep} B$, is as yet 
unclear. In $\mathrm{pWVO} 2$, the putative ORF $\mathrm{X}$ product will be truncated since a stop codon is present after codon 14 , which is followed by a transcriptional terminator within 100 bp (Kiewiet et al., 1993). No sequence errors are likely to be present in this region. The likely translational coupling of ORF $\mathrm{X}$ to $\operatorname{rep} B$ and the deduced DNAbinding properties of the ORF $X$ product suggest an involvement in replication. Since, however, ORF X is not present intact in pWVO2, and can be deleted from pJR7 without preventing replication, its presence is not essential.

Only a limited number of lactococcal plasmids did not hybridize to the $\mathrm{pWVO} 2$ rep $\mathrm{B}$ probe. A number of smaller plasmids hybridized with a probe derived from $\mathrm{pWVO} 1$ (results not shown). The latter RC replicon is also widespread among lactococci, but the strains tested so far by us and others (Dr A. Geis, personal communication) never contained more than one member of this family of plasmids. Although we have observed that a few plasmids do not hybridize to either probe (results not shown), and these might constitute yet another family of plasmids, the present results indicate that only two families of plasmid replicons are common in lactococci: the first family contains members of the pWVO2-type theta replicons, and the second, members of the pWVO1-type RC replicons.

\section{ACKNOWLEDGEMENTS}

Funding for the project was provided by the Dutch Program Commission for Industrial Biotechnology (Project number IOP-B 39103) and by Unilever Research, Vlaardingen, The Netherlands.

The lactococcal strains 9B4, P8-2-47, 916, 109, L10 and cremoris $\mathrm{Cla} 2$ were obtained through Dr Igor Mireau, who obtained them from the former 'Wissenschaftlich Technisches Oekonomisches Institut der Milchwissenschaft' of Oraniënburg (Germany).

\section{REFERENCES}

Bramhill, D. \& Kornberg, A. (1988). A model for initiation at origins of DNA replication. Cell 54, 915-918.

Bron, S., Meijer, W., Holsappel, S. \& Haima, P. (1991). Plasmid instability and molecular cloning in Bacillus subtilis. Res Microbiol 142, $875-883$.

Chopin, A., Chopin, M-C., Moillo-Batt, A. \& Langella, P. (1984). Two plasmid-determined restriction and modification systems in Streptococcus lactis. Plasmid 11, 260-263.

Exterkate, F. A. (1975). An introductory study of the proteolytic system of Streptococcus cremoris strain Hp. Neth Milk Dairy J 29, 303-318.

Frey, J., Bagdasarian, M. M. \& Bagdasarian, M. (1992). Replication and copy number control of the broad-host-range plasmid RSF1010. Gene 113, 101-106.

Gasson, M. J. (1983). Plasmid complements of Streptococcus lactis NCDO and other lactic streptococci after protoplast-induced curing. J Bacteriol 117, 283-289.

Gruss, A. \& Ehrlich, S. D. (1989). The family of highly interrelated single-stranded deoxyribonucleic acid plasmids. Microbiol Rev 53, 231-241.
Hayes, F., Vos, P., Fitzgerald, G. F., de Vos, W. M. \& Daly, C. (1991). Molecular organization of the minimal replicon of novel, narrow-host-range, lactococcal plasmid pCI305. Plasmid 25, 16-26.

Holo, H. \& Nes, I. F. (1989). High-frequency transformation, by electroporation, of Lactococcus lactis subsp. cremoris grown with glycine in osmotically stabilized media. Appl Environ Microbiol 55, 3119-3123.

Horng, J. S., Polzin, K. M. \& McKay, L. L. (1991). Replication and temperature-sensitive maintenance functions of lactose plasmid pSK11L from Lactococcus lactis subsp. cremoris. J Bacteriol 173, 7573-7581.

Jahns, A., Schäfer, A., Geis, A. \& Teuber, M. (1991). Identification, cloning and sequencing of the replication region of Lactococcus lactis ssp. lactis biovar. diacetylactis Bu2 citrate plasmid pSL2. FEMS Microbiol Lett 80, 253-258.

Kiewiet, R., Bron, S., de Jonge, K., Venema, G. \& Seegers, J. F. M. L. (1993). Theta replication of the lactococcal plasmid pWVO2. Mol Microbiol 10, 319-327.

Kiewiet, R., Kok, J., Seegers, J. F. M. L., Venema, G. \& Bron, S. (1993). The mode of replication is a major factor in segregational plasmid instability in Lactococcus lactis. Appl Environ Microbiol 59, 358-364.

Kok, J. (1991). Special-purpose vectors for lactococci. In Genetics and Molecular Biology of Streptococci, Lactococci and Enterococci, pp. 97-102. Edited by G. M. Dunny, P. P. Cleary \& L. L. McKay. Washington, DC: American Society for Microbiology.

Kok, J., Leenhouts, K., Haandrikman, A. J., Ledeboer, M. \& Venema, G. (1988). Nucleotide sequence of the cell wall proteinase gene of Streptococcus cremoris Wg2. Appl Environ Microbiol 54, 231-238.

Leenhouts, K. J., Tolner, B., Bron, S., Kok, J., Venema, G. \& Seegers, J. F. M. L. (1991). Nucleotide sequence and characterization of the broad-host-range lactococcal plasmid pWVO1. Plasmid 26, 55-66.

Mandel, M. \& Higa, A. (1970). Calcium-dependent bacteriophage DNA infection. J Mol Biol 53, 159-162.

Novick, R. (1989). Staphylococcal plasmids and their replication. Annu Rev Microbiol 43, 537-565.

Okamoto, T., Fujita, Y. \& Irie, R. (1983). Protoplast formation and regeneration of Streptococcus lactis cells. Agric Biol Chem 47, 259-263.

Okamoto, T., Fujita, Y. \& Irie, R. (1985). Interspecific protoplast fusion between Streptococcus cremoris and Streptococcus lactis. Agric Biol Chem 49, 1371-1376.

Otto, R., de Vos, W. M. \& Gavrieli, J. (1983). Plasmid DNA in Streptococcus cremoris Wg2: influence of $\mathrm{pH}$ on selection in chemostats of a variant lacking a protease plasmid. Appl Environ Microbiol 43, $1272-1277$.

Perri, S., Helinski, D. R. \& Toukdarian, A. (1991). Interactions of plasmid-encoded replication initiation proteins with the origin of DNA replication in the broad host range plasmid RK2.J Biol Chem 266, 12536-12543.

Sambrook, J., Fritsch, E. F. \& Maniatis, T. (1989). Molecular Cloning: a Laboratory Manual. Cold Spring Harbor, NY: Cold Spring Harbor Laboratory.

Sanger, F., Nicklen, S. \& Coulson, A. R. (1977). DNA sequencing with chain-terminating inhibitors. Proc Natl Acad Sci USA 74, 5463-5467.

Short, J. M., Fernandez, J. M., Sorge, J. A. \& Huge, W. D. (1988). ZAP: a bacteriophage expression vector with in vivo excision properties. Nucleic Acids Res 16, 7583-7600.

Terzaghi, B. E. \& Sandine, W. E. (1975). Improved medium for 
lactic streptococci and their bacteriophages. Appl Microbiol 29, 807-813.

Van de Guchte, M., Kok, J. \& Venema, G. (1992). Gene expression in Lactococcus lactis. FEMS Microbiol Rev 88, 73-92.

Visser, S., Exterkate, F. A., Slangen, C. J. \& de Veer, G. J. C. M. (1986). Comparative study of action of cell wall proteinases from various strains of Streptococcus cremoris on bovine $\alpha \mathrm{s} 1-, \beta$ - and $\kappa$ casein. Appl Environ Microbiol 52, 1162-1166.

Von Wright, A., Wessels, S., Tynkknen, S. \& Saarela, M. (1990).
Isolation of a replication region of a large lactococcal plasmid and use in cloning of a nisin resistance determinant. Appl Environ Microbiol 56, 2029-2035.

Yanisch-Perron, C., Vieira, J. \& Messing, J. (1985). Improved M13 phage cloning vectors and host strains: nucleotide sequences of M13mp18 and pUC19 vectors. Gene 33, 103-119.

Received 3 August 1993; revised 2 December 1993; accepted 20 December 1993. 